\title{
Diseño de tarjeta electrónica genérica para el control de motores trifásicos
}

\section{Generic Electronic Board Design to Control 3-Phase Ac}

\author{
Rojas-Molina A. \\ Facultad de Ingeniería \\ Universidad Autónoma de Querétaro, Querétaro \\ Correos:arojas@uaq.mx,adriana.rojasmolina@gmail.com \\ Herrera-Ruiz G. \\ Facultad de Ingeniería \\ Universidad Autónoma de Querétaro, Querétaro \\ Correo:gherrera@uaq.mx \\ Castañeda-Miranda R. \\ Universidad Politécnica del Sur de Zacatecas, Zacatecas \\ Correos:upsz.rectoria@gmail.com \\ Terrazas-Lara A. \\ Instituto Tecnológico de Chihuahua, Chihuahua \\ Correo:atl_trota_mundo@hotmail.com
}

\author{
Flores-Chaparro J.H. \\ Instituto Tecnológico de Chihuahua, Chihuahua \\ Correo:hissarlikbond@hotmail.com \\ Barón-Rodríguez B. \\ Facultad de Ingeniería \\ Universidad Autónoma de Baja California, Mexicali \\ Correo:benjamin.baron@descoa.com \\ Chaparro-Sánchez R. \\ Facultad de Ingeniería \\ Universidad Autónoma de Querétaro, Querétaro \\ Correo:rchapa@uaq.mx
}

Infomación del artículo: recibido: febrero de 2008, reevaluado: agosto de 2009 y agosto de 2010, aceptado: noviembre de 2010

\section{Resumen}

En este trabajo se presenta el diseño y desarrollo de un manejador de potencia con controlador para un motor trifásico de costo reducido y con una amplia gama de aplicaciones en la industria. El diseño y construcción del prototipo del manejador se realizó empleando el estándar genérico para diseño de tarjetas impresas IPC2221. El manejador propuesto se compone de dos etapas, la primera es la etapa de control en la que se incluye micro-controlador de función específica para control de motores, y la segunda es la etapa de potencia manejada con transistores de compuerta aislada (IGBT) como elementos de conmutación. Esta propuesta forma parte de un proyecto, que tiene como principal objetivo proponer una metodología que coadyuve a mejorar la calidad en los prototipos electrónicos que se desarrollan en ambientes académicos; de manera que estén más acordes con los estándares que exige la industria, facilitando la vinculación universidad-industria, en lo que respecta a la colaboración tecnológica en el ámbito de la automatización y el control.

\section{Descriptores}

- manejador de potencia

- metodología

- calidad

- prototipos

- estándares industriales

- motor de inducción 


\begin{abstract}
This work presents the design and development of a low-cost power manager with controller for a 3-phase AC induction motor, with a wide range of industrial applications. The prototype drive design and development was made using the generic standard on printed board design IPC2221. Power manager consists of two stages. First is control stage, which includes an induction motor control-specific micro-controller. Second is power stage, with IGBTs as switching elements. This design is part of a wider project whose main objective is to advance a methodology to assist the quality improvement of academia-developed electronic prototypes. It is expected that these prototypes will be aligned with industrial standards, facilitating the industryuniversity connection regarding technological collaboration in the automation and control domain.
\end{abstract}

\section{Introducción}

La vinculación de las universidades y centros de investigación y desarrollo con el sector productivo, es un tema que tiene más de una década de estar presente en México en los esquemas y planes de desarrollo económicos y sociales propuestos por distintos gobiernos tanto federales como estatales. La meta de esta vinculación es incorporar y aplicar el conocimiento científicotecnológico en la producción industrial para que éste sea un eje central de la competitividad de las empresas.

Sin embargo, a la fecha existen limitantes de las universidades en Latinoamérica para transferir desarrollos tecnológicos a la industria. Algunas de estas limitantes son la insuficiente capacidad de investigación para ofrecer soluciones tecnológicas a los problemas de las empresas manufactureras y el desconocimiento de los requisitos de calidad que deben cumplir un desarrollo tecnológico para llegar a ser competitivo a nivel industrial (Solleiro, 2008).

Existen a nivel académico diversos proyectos de investigación y desarrollo de tecnología relacionados con los motores eléctricos trifásicos. En los proyectos desarrollados por universidades latinoamericanas, con frecuencia se opta por la inclusión de controladores comerciales, tales como los controladores lógico programables (PLC), debido a que son ampliamente conocidos y confiables (Carabali et al., 2007). No obstante, esta solución no es viable para ser implementada en aplicaciones cuyo objetivo es la producción en serie a un bajo costo.

Entre las soluciones propuestas por la academia para el control más efectivo y adecuado de este tipo de motores, se encuentran desde control robusto y redes neuronales hasta control difuso, así como diseños que emplean software como MATLAB o PSPICE. Sin embargo, los prototipos de tarjetas electrónicas que se reportan, no son descritos con detalle, por ejemplo véase Egaña et al. (2001), Chamorro et al. (2010), Fonseca y Afonso (1999), Villada y Cadavid (2007), Marcos y Fernández (1996), Reyes (2009), Morales y Vanegas (2005), Tzou y Ling (1998), y Shi y Chan (2002). En los casos en que se reporta el desarrollo de prototipos de tarjetas de circuitos impresos (PCB), no se menciona si se aplican normas técnicas de empleo generalizado en la industria, que potencialmente facilitarían la transferencia de este tipo de soluciones a las empresas interesadas en emplearlas, véase Andrago et al (2000), Escobar et al (2005), Blancas et al (2007), Uddin (2005) y Nuñez (2009).

Inclusive en materiales integrados con fines didácticos a nivel licenciatura, no se hace mención de considerar estándares y normas técnicas durante el desarrollo y diseño de los PCB propuestos. Por ejemplo, Sandoval et al. (2006), Luna y Moctezuma (2004) no establecen si se tomaron previsiones para evitar la interferencia electromagnética en los circuitos.

Comparativamente, la revisión del estado de la cuestión encontró un número pequeño de casos de instrumentación exitosa en propuestas de origen académico. En uno de éstos, (Badilla, 2002), es notorio el empleo de un controlador comercial, con un costo 3.5 veces mayor que el del motor empleado.

Asimismo, existen proyectos formales encaminados a reducir los costos y aumentar la efectividad de los controladores electrónicos para motores trifásicos, mediante la eliminación del uso de sensores para llevar a cabo el control del motor, véase Salas et al.(2006) y Bisheimer et al. (2004); el diseño de esquemas de control más efectivos para convertidores $\mathrm{AC} / \mathrm{DC} / \mathrm{AC}$ de una fase a tres fases, mediante Modulación por Ancho de Pulso (PWM) utilizando un procesador digital de señales (DSP) (Lee y Kim, 2007), o el empleo de Arreglos de Compuertas Programables (FPGA) en combinación con un DSP para proveer una solución efectiva para controladores AC de alto desempeño, tal y como proponen 
Tzou y Hsu (1997). Cabe destacar que en estos trabajos no se describen las consideraciones que se tomaron para el desarrollo de los prototipos PCB propuestos. En el trabajo de Tzou y Hsu, sería interesante realizar un análisis de costo que compruebe que esta solución representa una opción viable para la producción en masa.

El presente trabajo tiene su origen en la petición de una empresa de electrodomésticos, para desarrollar un prototipo de manejador de potencia con controlador para un motor trifásico. Derivado de la revisión del estado del arte, se consideró oportuno emplear metodologías que garantizaran la satisfacción de los requerimientos del cliente.

La metodología utilizada se basa en la conceptualización del proceso de diseño y construcción del prototipo electrónico, bajo un esquema de interacción coordinada entre el controlador (software de control) y el subsistema controlado (la planta) (Von, 2007). Además se emplea como herramienta principal para el diseño de la tarjeta electrónica, el "Estándar Genérico para el Diseño de Tarjetas Impresas 2221" IPC2221 (1998), con el fin de cumplir con normas básicas de diseño y producción electrónica.

\section{Requerimientos del cliente}

El cliente requiere controlar un motor trifásico en su aplicación. Este tipo de motores requieren de poco mantenimiento, además de que son demandados por un mercado que exige cada vez más electrodomésticos con mejor desempeño.

El control de motores trifásicos en conjunto con la aplicación del cliente, requiere de algoritmos de control sofisticados, cuya implementación se lleva a cabo a través de un micro-controlador que permite un procesamiento de señales en tiempo real. El controlador de motor trifásico requerido por el cliente debe controlar la velocidad de un motor de inducción trifásico AC, tener protección de sobre-voltaje y sobre-corriente y debe contener retroalimentación de corriente para llevar a cabo el control del motor.

\section{Desarrollo}

Para cumplir con los requerimientos genéricos de diseño y construcción de tarjetas electrónicas, se utilizó del estándar IPC2221 (desarrollado por el Institute for Interconnecting and Packaging Electronic Circuits) aquellas recomendaciones y requerimientos que son aplicables al prototipo propuesto, dejando de lado aquellas que no aplicaban tanto por la naturaleza del prototipo, como por los materiales empleados.
El estándar IPC2221, establece principios de diseño y recomendaciones que deben ser utilizados en conjunto con los requerimientos específicos de cada aplicación.

De acuerdo al estándar IPC2221, la tarjeta propuesta pertenece a la Clase 1 (productos electrónicos generales) en los cuales el principal requerimiento es el funcionamiento de la tarjeta impresa ensamblada, mientras que el aspecto cosmético no es importante.

El software de diseño electrónico utilizado para diseño del esquemático y el diseño de tarjeta fue el Design Explore 99 SE. El maquinado de la tarjeta se realizó con la herramienta ProtoMat C60.

Conforme a IPC2221, el éxito en el diseño de una tarjeta de circuito impresa depende de diversos factores:

- las condiciones de trabajo de la tarjeta: temperatura ambiente y calor generado por los componentes,

- la facilidad de reparación y mantenimiento, ésta consideración incide directamente en la densidad de los circuitos y la posición de los componentes,

- los requerimientos para realizar pruebas que pueden afectar la posición de los componentes, el ruteo de pistas y la asignación de conectores,

- determinadas consideraciones pare evitar la interferencia electromagnética (EMI).

Adicionalmente, es importante considerar la existencia de un producto previo y los requerimientos del cliente de manera que se pueda mejorar el tiempo de entrega y el desempeño del PCB a diseñar y construir. Para el prototipo del manejador propuesto se analizaron y aplicaron las siguientes consideraciones del estándar IPC2221.

\section{Consideraciones de diseño}

El diseño de las características físicas de una tarjeta, así como la selección de los materiales de un PCB, requiere de un balance entre el desempeño mecánico, eléctrico y térmico, además de un balance entre confiabilidad, manufactura en masa y costo de la tarjeta.

Existen algunas consideraciones muy simples para reducir el tiempo y costo de una búsqueda de defectos. Por ejemplo, la orientación de las partes o componentes polarizados debe ser consistente, de manera que el diseñador no confunda componentes orientados $180^{\circ}$ fuera de fase con respecto a otros. Los componentes no polarizados deben tener plenamente identificado la terminal (pin) \#1, de forma que el operador que realiza las pruebas tenga referencia sobre qué pin específico es el que debe ser probado. 
En el diseño de un PCB, es recomendable una separación de funciones con el objeto de mejorar el desempeño eléctrico del PCB. Por ello, para este prototipo desde el planteamiento lógico y esquemático se sugirió la integración de dos etapas, la etapa de control cuya principal tarea es proporcionar la base para la ejecución de los algoritmos de control de velocidad y par del motor, así como la etapa de potencia cuya tarea principal es acondicionar las señales que irán directamente al motor, conforme al algoritmo de control utilizado. Asimismo, dados los requerimientos de tamaño de la tarjeta y las consideraciones de ambiente de trabajo, facilidad de mantenimiento y reparamiento y costos de producción se planteó el diseño de un prototipo de 2 capas. En la figura 1 se ilustra el diagrama a bloques del manejador.

\section{Consideraciones eléctricas}

Un factor muy importante que debe considerarse en el diseño de un PCB es la distribución de la alimentación eléctrica. Un buen esquema de tierra proporciona un retorno de alimentación de corriente directa (DC), y adicionalmente un plano de referencia para las señales de corriente alterna (AC) de alta velocidad. Por el contrario, un esquema de tierra mal diseñado puede generar impedancia de frecuencia baja a través de la alimentación DC, como resultado de los diferentes campos generados por impedancias desbalanceadas y la incapacidad de los capacitores de desacoplamiento para reducir de manera eficiente las interferencias electromagnéticas (EMI).

Un desacoplamiento adecuado de la alimentación eléctrica se lleva a cabo a través de una distribución uniforme de capacitores de desacople en todas las zonas del PCB. Por otro lado, el lazo de impedancia de un capacitor de desacople se minimiza manteniendo sus conectores lo más pequeños posible y colocándolos en un encadenamiento margarita en el orden correcto, cercano al circuito de interés (figura 2).

Por otro lado, se recomienda que la asignación de las señales a las terminales de un dispositivo tengan referencia a la señal de tierra (Martínez, 2010).

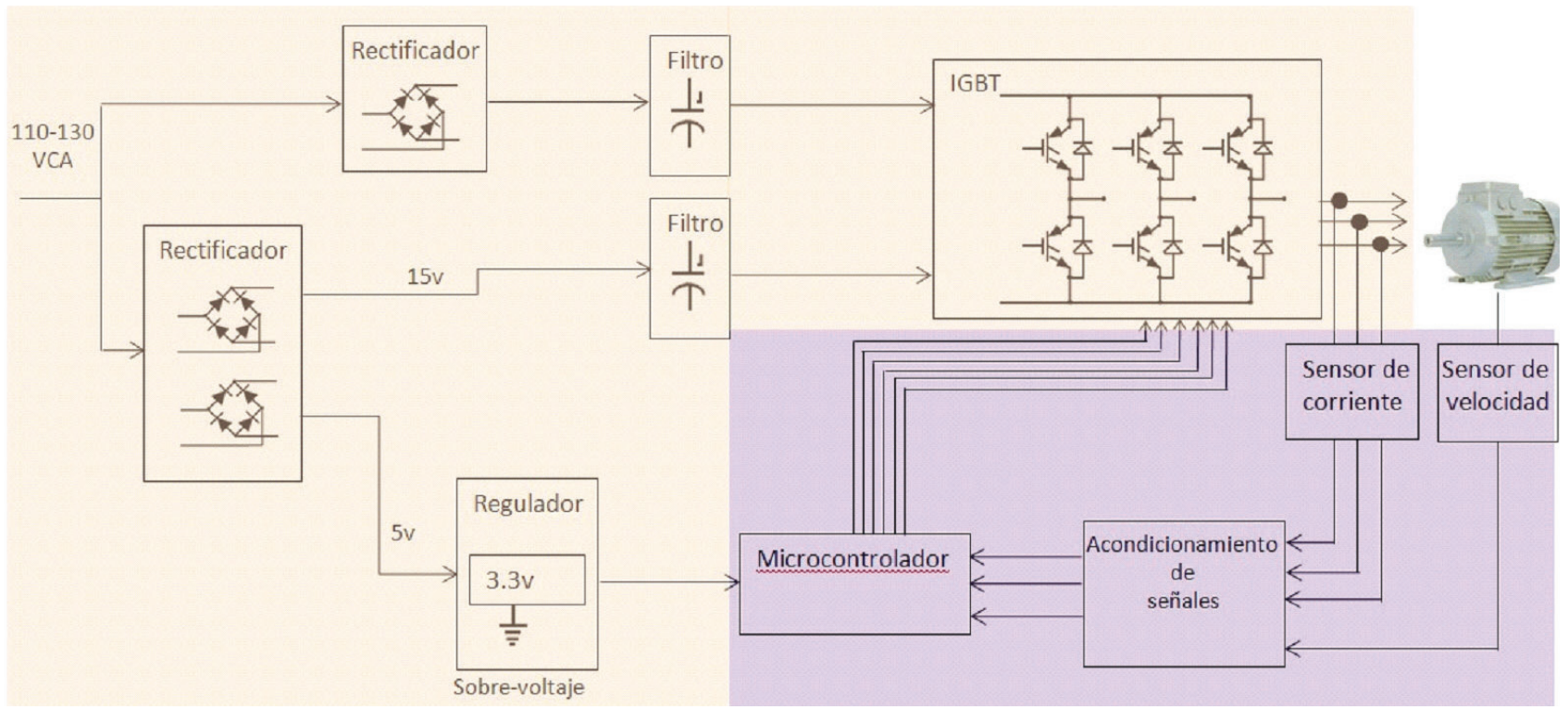

Figura 1. Esquema a bloques del prototipo propuesto

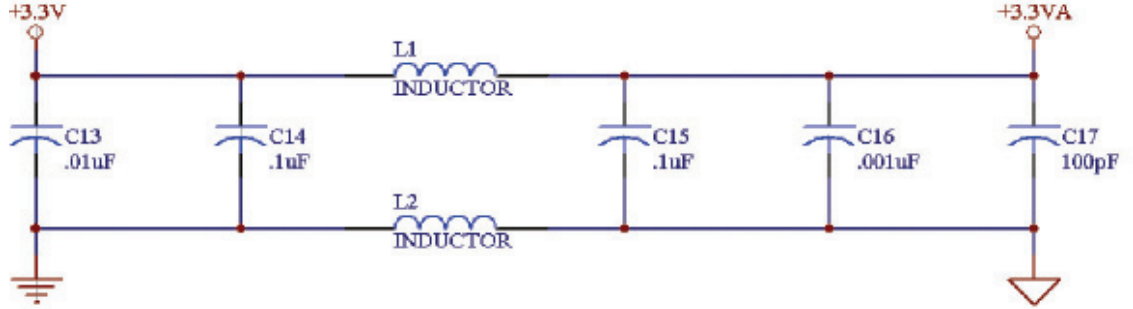

Figura 2. Capacitores conectados en margarita para filtrado de la señal de alimentación de microprocesador 


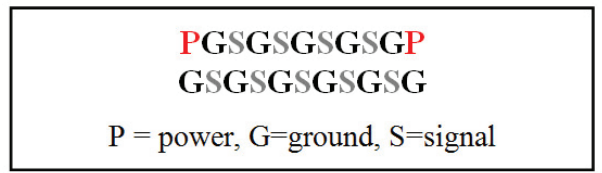

(a)

\begin{tabular}{c} 
SSSGSSSGSSP \\
SGSSSGSSSGP \\
$\mathrm{P}=$ power, $\mathrm{G}=$ ground, $\mathrm{S}=$ =signal \\
\hline
\end{tabular}

(b)

Figura 3. Asignación de señales a los pines de un componente electrónico

En la figura 3a se muestra una asignación de señales ideal. No obstante, esta solución no es práctica ni económica, de manera que una solución más adecuada es la que se ilustra en la figura $3 b$, donde cada señal está espaciada por una terminal de la señal de tierra. Otra consideración a tomar en cuenta es priorizar las señales colocando aquellas que son críticas adyacentes a las señales de tierra y las de menor prioridad con una terminal de separación de la señal de tierra.

\section{Consideraciones en los componentes}

Algunas de las consideraciones con respecto a los componentes empleados en la tarjeta electrónica incluyen:

- determinar la polaridad correcta de cada componente,

- mantener las rutas entre los componentes lo más pequeñas posible,

- $\quad$ si se utilizan diferentes señales de tierra, se deben mantener las rutas o planos de estas señales lo más lejanas posible,

- mantener los componentes que son afectados por el calor y los componentes generadores de calor lo más separados posible, incorporando enfriadores (heatsinks) cuando sea necesario.

\section{Consideraciones de separación entre pistas}

La separación entre pistas sobre capas individuales debe ser la máxima posible. El espacio mínimo entre pistas y componentes montados en la tarjeta está definido en el estándar conforme a los picos de voltaje presentes en las pistas, si es una pista interna o externa y la altura sobre el nivel del mar. Para el manejador propuesto, se utilizó una separación de 50 milésimas de pulgada $(0.127 \mathrm{~cm})$ entre pistas y 100 milésimas de pul- gada $(0.254 \mathrm{~cm})$ entre componentes, que es mayor que el mínimo establecido para conductores y componentes externos, sin aislamiento y hasta una altura de $3050 \mathrm{~m}$.

\section{Descripción de la etapa de control}

Para el diseño de la etapa de control se consideró la inclusión de un micro-controlador MC56F8013, fuentes de alimentación de $5 \mathrm{~V}$ y $3.3 \mathrm{~V}$, una interfaz serial RS-232 con conector DB9, una interfaz IEEE 1149.1 (Joint Test Action Group, JTAG) para emulación del micro-controlador y una interfaz con la etapa de potencia a través de conector de 26 terminales.

El micro-controlador MC56F8013, consta de un módulo de modulación de ancho de pulso (PWM) con 6 salidas, convertidores ADC de 12 bits que permiten conversiones simultáneas y una interfaz serial.

La programación del algoritmo de control, así como las pruebas de software, se realizaron a través de la interfaz IEE1149.1 (figura 4) empleando el software CodeWarrior.

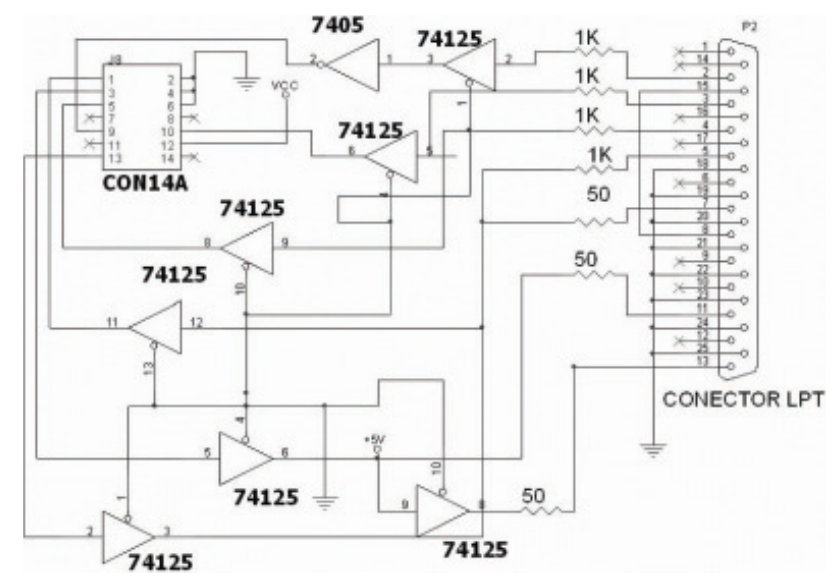

Figura 4. Diagrama esquemático de la interfaz IEEE 1149.1

\section{Descripción de la etapa de potencia}

Para el diseño de esta etapa se incluyeron: un convertidor de fuente de voltaje formado por 6 transistores de compuerta aislada (IGBT), un acondicionador de señales para retroalimentación de corrientes, un sensor de velocidad y fuentes de voltaje de $170 \mathrm{~V}$ y $15 \mathrm{~V}$. El componente principal de esta etapa es el convertidor de fuente voltaje. La tabla 1, muestra algunas de las características eléctricas del IGBT empleado (IRAMS10UP60). En la figura 5 se muestra la conexión del IGBT con el microprocesador, la retroalimentación de corriente para llevar a cabo el control del motor y las salidas de PWM. Asimismo, se observó la conexión del IGBT con el motor trifásico. 
Tabla 1. Características eléctricas del IGBT

\begin{tabular}{|c|c|c|c|}
\hline Parámetro & Descripción & $\begin{array}{l}\text { Valor } \\
\text { máximo }\end{array}$ & Unidades \\
\hline $\mathrm{V}_{\mathrm{CES}}$ & $\begin{array}{l}\text { Voltaje máximo de bloqueo } \\
\text { IGBT }\end{array}$ & 600 & \multirow[b]{2}{*}{ V } \\
\hline $\mathrm{V}^{+}$ & $\begin{array}{l}\text { Voltaje positivo de bus de } \\
\text { entrada }\end{array}$ & 450 & \\
\hline $\mathrm{I}_{0} @ \mathrm{~T}_{\mathrm{c}}=25^{\circ} \mathrm{C}$ & Corriente en fase RMS & 10 & \multirow{3}{*}{ A } \\
\hline $\mathrm{I}_{0} @ \mathrm{~T}_{\mathrm{c}}=100^{\circ} \mathrm{C}$ & Corriente en fase RMS & 5 & \\
\hline $\mathrm{I}_{\mathrm{pk}}$ & $\begin{array}{l}\text { Máximo pico de corriente en } \\
\text { fase }(\mathrm{tp}<100 \mathrm{~ms})\end{array}$ & 15 & \\
\hline $\mathrm{F}_{\mathrm{p}}$ & $\begin{array}{l}\text { Máxima frecuencia de origen } \\
\text { del PWM }\end{array}$ & 20 & $\mathrm{kHz}$ \\
\hline$P_{d}$ & $\begin{array}{l}\text { Máxima potencia disipada } \\
\text { por fase }\end{array}$ & 20 & W \\
\hline $\mathrm{V}_{\text {iso }}$ & Voltaje de aislamiento $(1 \mathrm{~min})$ & 2000 & $\mathrm{~V}_{\mathrm{RMS}}$ \\
\hline VCC & $\begin{array}{l}\text { Voltaje de alimentación } \\
\text { principal }\end{array}$ & 15 & $\mathrm{~V}$ \\
\hline
\end{tabular}

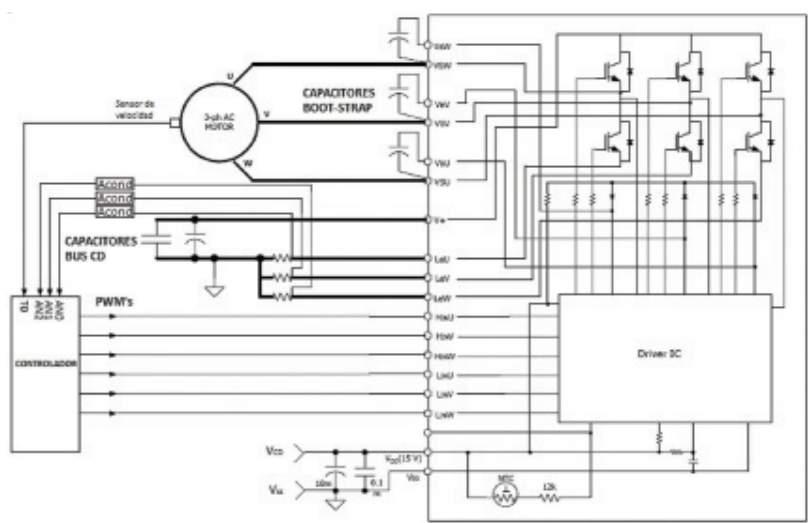

Figura 5. Conexión del controlador de voltaje IGBT

También se puede observar en la figura 5 que la señal de retroalimentación de corriente obtenida a través de resistencias tipo Shunt, pasa por un módulo de acondicionamiento para ser amplificada y convertida a una señal digital. La configuración del módulo de acondicionamiento es mostrado en la figura 6.

Las señales PWM generadas por el micro-controlador son alimentadas directamente al módulo IGBT. No se implementó ningún tipo de aislamiento, ya que la inclusión de éstos elevaría el costo de producción en masa del prototipo. Cabe mencionar que si el módulo IGBT hiciera cortocircuito, el costo-beneficio de proteger el microprocesador no es equiparable a la pérdida del módulo IGBT, es decir, no tendría caso proteger el microprocesador ya que la relación de costo entre éste y el IGBT es del orden de 1 a 10, siendo el IGBT el componente más caro de la tarjeta.

Los requerimientos del cliente establecen que no es necesario conocer el sentido del giro, sino únicamente asegurar un cambio de éste y que en la aplicación final

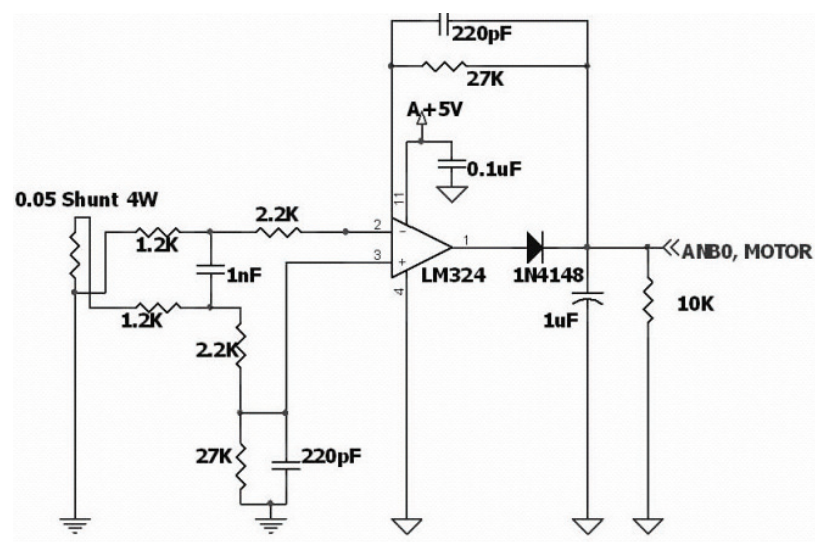

Figura 6. Diagrama esquemático del módulo de acondicionamiento

se emplee un sensor de efecto hall para conocer la velocidad del motor.

Para satisfacer los requerimientos del cliente en el sentido antes mencionado, se diseñó un sensor de velocidad empleando un led infrarrojo y un fototransistor, cuya señal es convertida a señal digital a través de un comparador LM324 (figura 7).

Para la alimentación de 15V (requerida por el convertidor de voltaje, IGBT) y la alimentación de 5V (requerida por la etapa de control) se proponen dos soluciones, la primera de ellas involucra el diseño de una fuente conmutada implementada a través de un convertidor de energía Viper22A, la segunda solución implica el diseño de una fuente con transformador.

En la tabla 2 se muestran las ventajas y desventajas de cada una. En el prototipo propuesto se eligió el uso del transformador por la ventaja del aislamiento de línea y para efectos de prueba; sin embargo, para trabajos futuros se propone el uso del Viper22A por razones de costo.

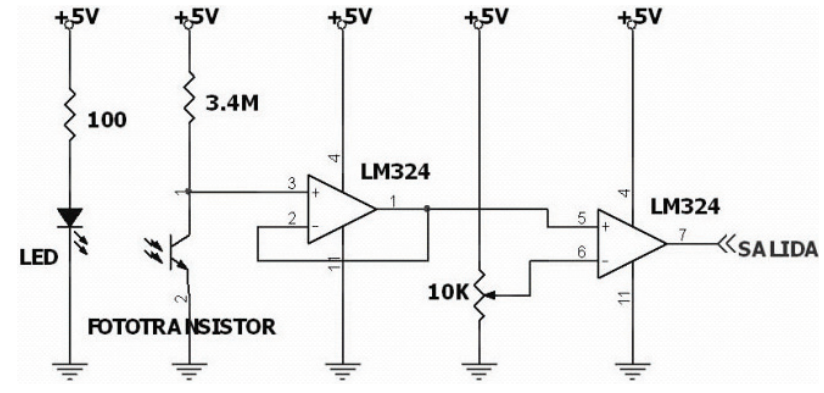

Figura 7. Diagrama esquemático del sensor de velocidad 
Tabla 2. Comparación entre ambas opciones

\begin{tabular}{cc}
\hline \multicolumn{2}{c}{ Usando Viper22A } \\
\hline $\begin{array}{c}\text { Ventajas } \\
\text { Tanaño reducido } \\
\text { Menor costo }\end{array}$ & $\begin{array}{c}\text { Desventajas } \\
\text { No hay tierra fija, debido a la } \\
\text { conmutación entre Fase y Neutro. }\end{array}$ \\
Usando transformador \\
Aislamiento de línea & $\underline{\text { Desventajas }}$ \\
& Tamaño grande \\
& Mayor costo \\
\hline
\end{tabular}

\section{Pruebas}

Las pruebas del PCB propuesto se llevaron a cabo por funciones. En la parte control se realizaron:

- Verificación de los voltajes de alimentación del micro-controlador, los cuales deben mantenerse de manera continua dentro del rango de trabajo (3.3 a 3.6V) especificado por el fabricante.

- Verificación de las señales PWM generadas por el microcontrolador, para lo cual se realizaron:

- verificación del desfasamiento de señales PWM.

- verificación de la programación de tiempos muertos para evitar que los transistores de una misma rama estén encendidos simultánea mente.

Para la etapa de potencia se realizaron:

- Verificación de voltajes de alimentación de $15 \mathrm{~V}, 5 \mathrm{~V}$ y $170 \mathrm{~V}$, ya que se considera de suma importancia garantizar que los voltajes de alimentación requeridos

a) Diagrama esquemático

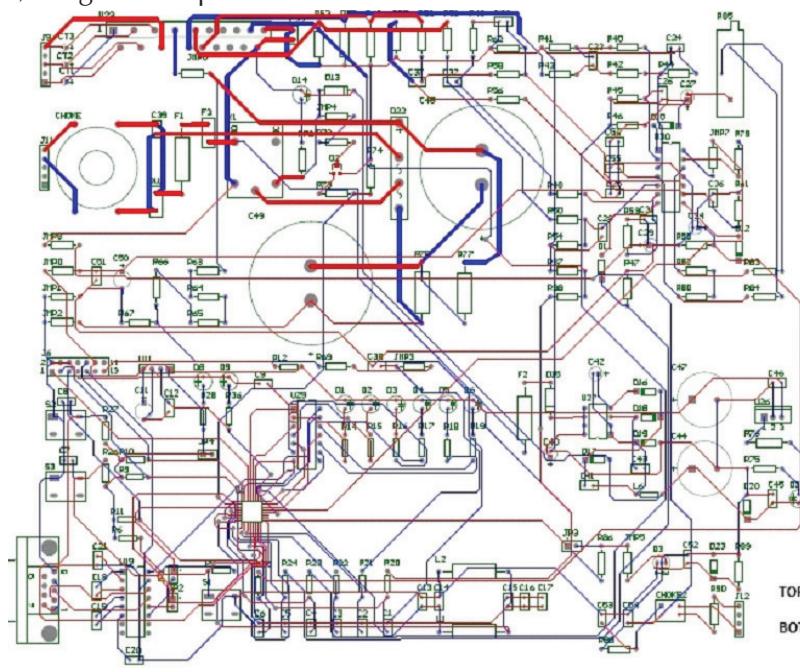

TOP LAYER BOTTOM LAYER por los módulos IGBT y acondicionador de señales, fueran confiables para garantizar un voltaje de alimentación dentro del rango de trabajo de los circuitos empleados.

- Verificación del funcionamiento del módulo IGBT, para lo cual se realizó:

- la verificación de la señal de voltaje trifásica proporcionada por el motor sin carga.

Las características del motor empleado para realizar las pruebas de la tarjeta se muestran en la tabla 3. Se utilizó un osciloscopio TDS 210 para realizar las mediciones de voltaje y verificar las formas de onda obtenidos.

Tabla 3. Características del motor de inducción

\begin{tabular}{cc}
\hline Especificación & Valor \\
\hline Frecuencia nominal & $50 \mathrm{~Hz}$ \\
Voltaje de línea nominal & $220 \mathrm{~V}$ \\
Número de polos & 2 \\
Factor de potencia & 0.79 \\
Corriente nominal & $1.4 \mathrm{~A}$ \\
Potencia nominal & $345 \mathrm{~W}$ \\
\hline
\end{tabular}

\section{Resultados}

El diseño de la tarjeta de dos capas se muestra en la figura 8.

Los voltajes de alimentación para la etapa de control y potencia se mantuvieron constantes y dentro de los rangos de trabajo deseado. La figura 9 muestra las lecturas y gráficas obtenidas por el osciloscopio para cada uno de los voltajes requeridos por los componentes del PCB.

b) Diagrama en 3D del diseño

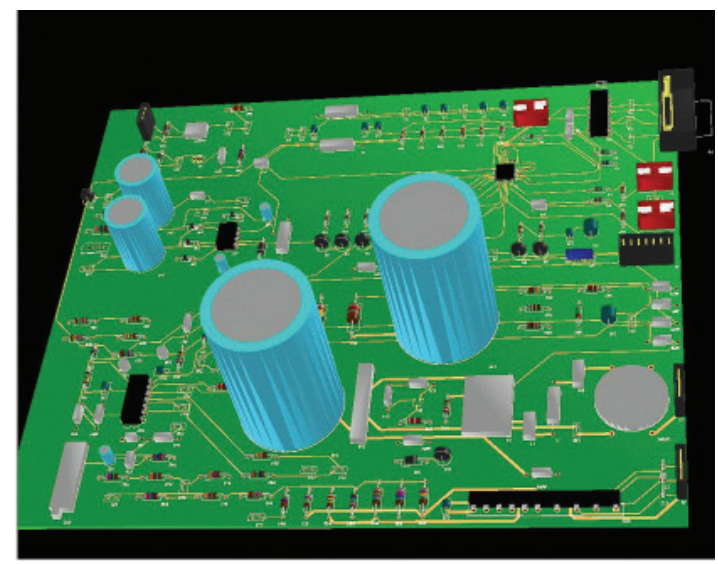

Figura 8. Diseño esquemático de prototipo propuesto 
a) fuente de $3.3 \mathrm{~V}$

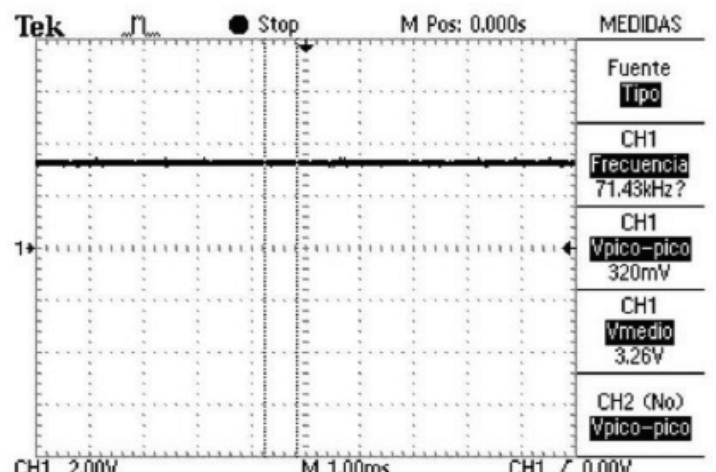

c) fuente de $15 \mathrm{~V}$

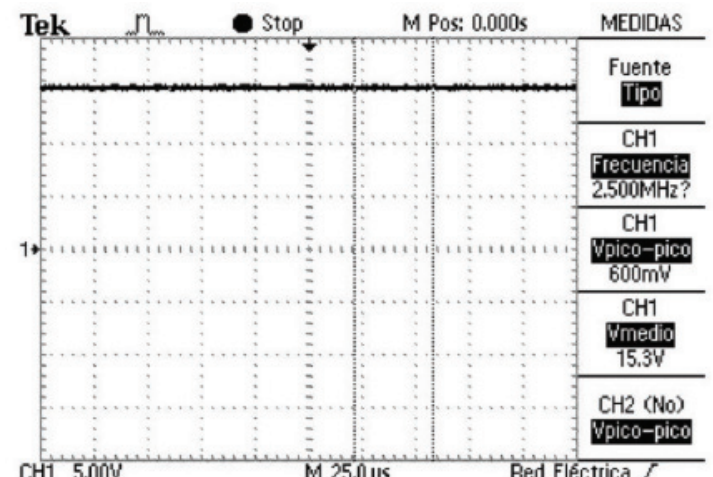

Figura 9. Mediciones de las fuentes de alimentación
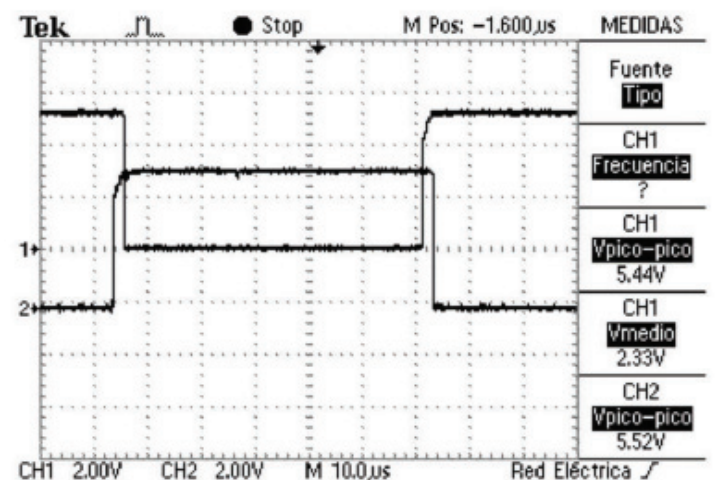

Figura 10. Salidas de microcontrolador para PWMO y PWM1 en canal A b) fuente de $5 \mathrm{~V}$

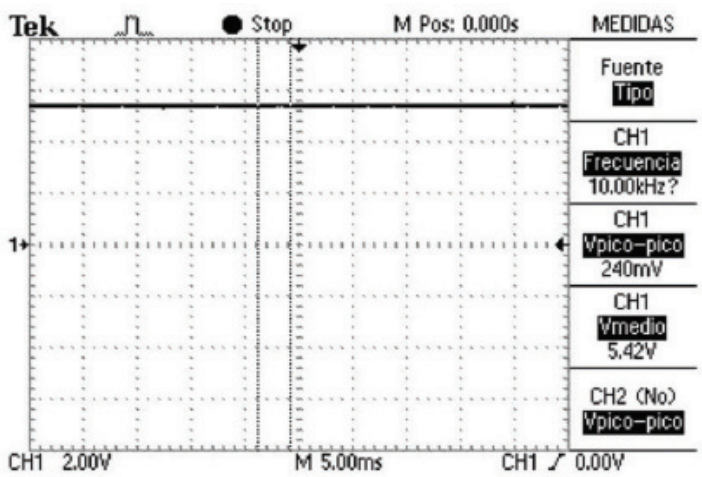

d) fuente de $170 \mathrm{~V}$

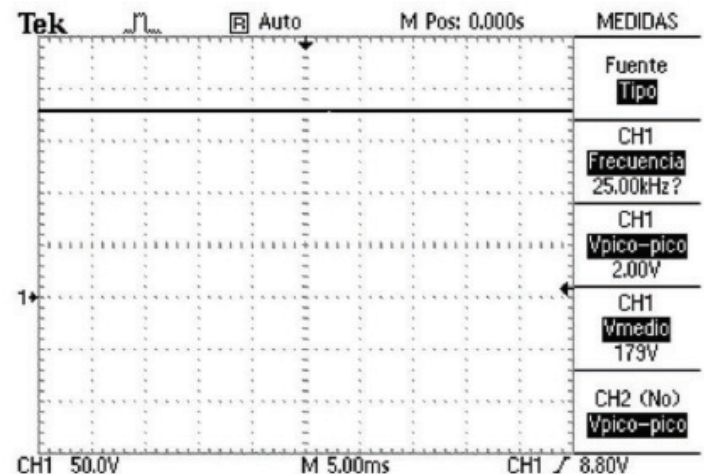

M 5 .

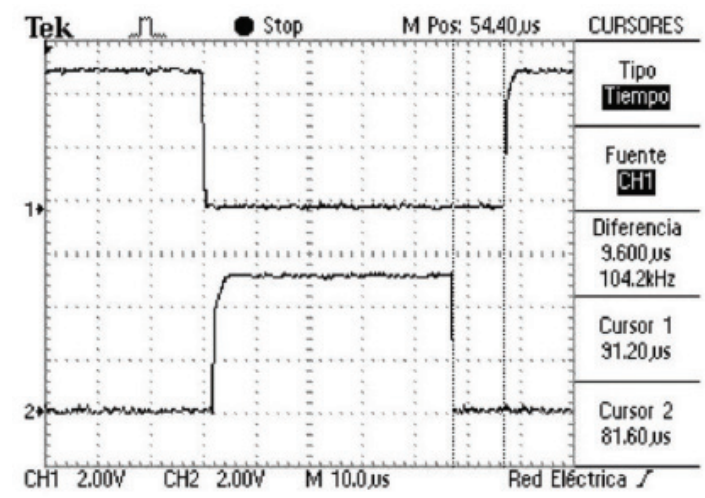

Figura 11. Tiempo muerto presente entre PW0 y PW1 en canal A 


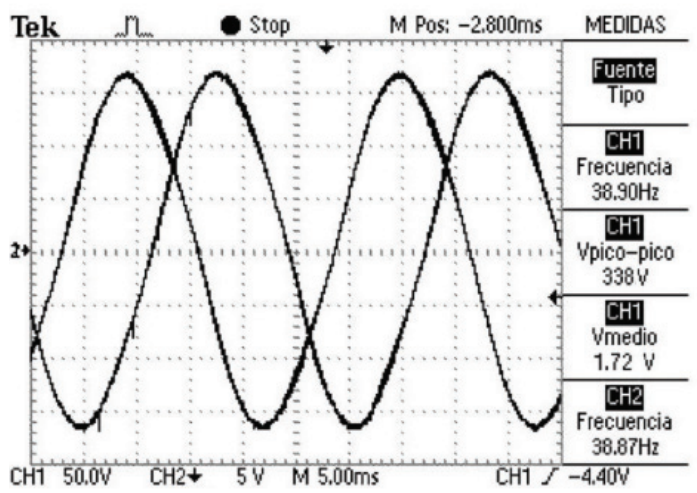

Figura 12. Señal trifásica con filtro pasabajas

Las salidas de las señales de PWM fueron consistentes con los requerimientos mínimos especificados por el fabricante del IGBT (figura 10).

El tiempo muerto programado fue de 10 microsegundos, el cual es mayor al recomendado mínimo de (300 ns), (figura 11).

Las formas de onda de la señal trifásica del motor sin carga (prueba al vacío) se muestran en la figura 12, donde se aprecian 2 fases. Se utilizó un filtro pasabajas para eliminar el efecto de los PWM y apreciar el desfasamiento presente en las señales sinusoidales.

Tabla 4. Relación frecuencia-velocidad, además de la corriente consumida

\begin{tabular}{ccc}
\hline Frecuencia $(\mathrm{Hz})$ & RPM & Corriente (Amp) \\
\hline 30 & 1786 & 2.46 \\
40 & 2377 & 1.92 \\
50 & 2954 & 1.88 \\
60 & 3554 & 2.14 \\
70 & 5263 & 2.6 \\
80 & 4477 & 3.16 \\
90 & 4961 & 3.82 \\
100 & 5631 & 4.72 \\
110 & 5861 & 6.1 \\
120 & 6138 & 7.5 \\
\hline
\end{tabular}

La relación entre frecuencia de la señal proporcionada y la velocidad del motor se muestra en la tabla 4.

\section{Conclusiones y trabajo futuro}

Se obtuvo un prototipo de manejador básico de potencia con controlador para un motor trifásico. Las pruebas realizadas estuvieron enfocadas principalmente a la verificación de las señales de la etapa de potencia y de control en lo que respecta a fuentes de alimentación y adecuación de señales de retroalimentación, con el objetivo de proporcionar una tarjeta confiable que pueda servir como base para prueba de algoritmos de control de motores de inducción más sofisticados, eliminando la incertidumbre de posibles fallas originadas por un mal diseño electrónico.

La relevancia del prototipo obtenido, radica en que su diseño y construcción se realizó aplicando un estándar industrial de uso extendido que permitirá, en un trabajo futuro, la aplicación de técnicas de aseguramiento de la calidad, la elaboración y realización de un plan de pruebas y verificación, con el cual será posible además detectar errores ocultos de diseño, funcionamiento y omisión en el cumplimiento de los requerimientos del cliente y la realización de propuestas de mejora en desempeño y costo de la tarjeta propuesta.

El uso de estándares hace posible, en una etapa posterior, la existencia de un plan de pruebas y verificación, así como una documentación adecuada en el desarrollo de prototipos elaborados en el ambiente académico. Lo anterior es importante no sólo porque facilita la transferencia de esta tecnología al minimizar la incertidumbre en la comunicación entre usuarios (empresas) y proveedores (investigador), sino porque permite determinar en términos medibles los niveles de seguridad, eficiencia y repetibilidad del prototipo.

Además de aumentar la confianza que los usuarios tienen en los prototipos que proporciona el sector académico, el empleo de una metodología formal capacita a los estudiantes de ingeniería en habilidades y capacidades que se requieren para impulsar la innovación tecnológica.

\section{Referencias}

Andrago M., Sotomayor L., et al. Simulación y ensamblaje de un prototipo para control y navegación de un robo móvil, en: Jornadas de ingeniería eléctrica y electrónica (18, 2000, Quito, Ecuador) [en línea]. Disponible en: http://dspace.epn.edu.ec/ handle/123456789/283

Badilla J. Sustitución del motor $\mathrm{CD}$ de una máquina extrusora tipo KMD2-90Z, por un motor CA y generación del módulo de control para la máquina [en línea]. Instituto Tecnológico de Costa Rica, 2002. Escuela de Ingeniería Electrónica. Disponible en: http://hdl.handle.net/2238/142

Bisheimer G., Sonnaillon M., et al. Estimación de estados en accionamientos eléctricos con reducción del número de sensores, en: AADECA 2004 XX Congreso Argentino de Control Automático $\left(20^{\circ}, 2004\right.$, Buenos Aires).

Blancas I., Contreras E., Martínez F. Control moderno aplicado a máquinas eléctricas rotatorias y sistemas automatizados. Tesis (Licen- 
ciatura). México. Escuela Superior de Ingeniería Mecánica y Eléctrica IPN. 2007. Pp. 24-37.

Carabali A., Almeida M. Diseño y construcción de un módulo automatizado didáctico para el control de arranque e inversión de giro de un motor de inducción trifásico y de un sistema de generación eléctrico monofásico. Tesis (Técnico Superior). Ecuador. 2007. Escuela Politécnica Nacional, Escuela de Tecnología. Pp. 90-103.

Chamorro H., Vladimir B., Trujillo C. Diseño y simulación de un controlador PD difuso para el control de la velocidad de un motor de inducción. Revista Científica Ingeniería y Desarrollo, Num. 27 [en línea]. Barranquilla, Colombia, 2010 [fecha de consulta: 5 de Agosto de 2010]. Disponible en: http://rcientificas.uninorte.edu.co/index.php/ingenieria/issue/view/54

Dong L., Young-Sim K. Control of Single-Phase-to-Three-Phase AC/DC/AC PWM Converters for Induction Motor Drives. IEEE Trans.on Industrial Electronics, 54(2):797-804, abril 2007.

Donner G.L., Subler W.L., Evon S.T. A Motor Primer-Part I. IEEE Transactions on industry applications, 36(5), September/October 2000.

Egaña I., Mota J., Castillejo J. Control robusto QFT de un helicóptero de 3 GDL, en: Jornadas de Automática (XXII, 2001 Bárcelona, España). Comité Español de Automática, España.

Escobar C., Martínez J., Telléz G. Control de un motor Brushless DC con frenado regenerativo. Tesis (Licenciatura). Colombia. Pontificia Universidad Javeriana. 2005. Pp. 42-55.

Fonseca J., Afonso J., et al. Evaluación de la aplicación de técnicas de lógica difusa en el control de máquinas eléctricas. Información Tecnológica, 1999 [en línea]. [Fecha de consulta: 5 Agosto de 2010], La Serena, Centro de Información Tecnológica. Disponible en: http://hdl.handle.net/1822/1747.

Luna G., Moctezuma G., et al., Diseño didáctico de convertidores CD-CA (a): Inversor monofásico, en: Congreso interuniversitario de electrónica, computación y eléctrica, Guadalajara, México, 2004. [Fecha de consulta: 16 Mayo 2010]. Disponible en: http://galia.fc.uaslp.mx/lic/P1_09a.pdf

Marcos J., Fernández A. Modelizado y simulación del control electrónico de motores con PSPICE, en: Congreso TAEE- Tecnologías Aplicadas a la Enseñanza de la Electrónica, (II, 1996, Sevilla). Sevilla, España. Universidad Politécnica de Madrid [en línea]. [Fecha de consulta: 2 Mayo 2010]. Disponible en: http://www3.euitt.upm.es/taee/Congresosv2/1996/ papers/1996S1D02.pdf

Martinez T. Effective PCB Design: Techniques to improve performance, en: Webinar técnicas para mejorar el desempeño y robustez a eventos de EMC, 2010 [en línea]. Disponible en: http://www.freescale.com/webapp/sps/site/training_information.jsp?code=WBNR_LA2010_EMC_SPANISH

Monti A., Santi E., Dougal R., Riva M. Rapid Prototyping of Digital Controls for Power Electronics, 18(3), 2003.

Morales D., Vanegas J. Diseño de un controlador de velocidad para un motor de inducción utilizando control vectorial. Tesis (Licenciatu- ra). Universidad del Cauca, Facultad de Ingeniería Electrónica y Telecomunicaciones. Colombia, Popayán. 2005. Pp. 91-120.

Motto. Gate Drive Techniques for Large IGBT Modules, Powerex Inc., PCIM, 1997, p. 1.

Nuñez-Gutierrez C., Lira-Perez J., et al. Control de un rectificador PWM con funciones ampliadas basado en un procesador digital de señales. Ingeniería, investigación y tecnología, 10(1), 2009.

Reyes L. Diseño e implementación de un programa basado en redes neuronales para el control de máquinas trifásicas de inducción. Tesis (Licenciatura). Escuela Politécnica Nacional, Facultad de Ingeniería Eléctrica y Electrónica. Ecuador. 2009.Pp. 155-160.

Salas P.O., De León M.J. Controles por retroalimentación de salida para motor de inducción sin sensores mecánicos. Ciencia UANL, IX(004), 2006.

Sandoval J., Salamanca W, et al. Desarrollo de un inversor monofásico didáctico, en: Congreso TAEE- Tecnologías Aplicadas a la Enseñanza de la Electrónica, (VII, 2006, Madrid). Madrid, España, Universidad Politécnica de Madrid [en línea]. [Fecha de consulta: 20 Agosto 2010]. Disponible en: . http://www3.euitt.upm.es/taee/Congresosv2/2006/ papers/2006S1G03.pdf

Shi K.L., Chan T.F., et al. Speed Estimation of an Induction Motor Drive Using an Optimized Extended Kalman Filter. IEEE Trans. on Industrial Electronics, 49(1), 2002.

Solleiro R.J.L [en línea]. [fecha de consulta: 9 de mayo de 2008]. Disponible en: http: //www.slideshare.net/bemaguali/vinculación-universad-empresa

The Institute for Interconnecting and Packaging Electronic Circuits, IPC-2221 Generic Standard on Printed Board Design, 1998

Tzou Ying, Hsu-Hau-J. FPGA Realization of Space-Vector PWM Control IC for Three Phase PWM Inverters. IEEE Trans. On Power Electronics,12(6):953-963, noviembre 1997.

Tzou Ying-Yu, Ling Shiu-Yung. Fuzzy-Tuning Current-Vector Control of a Three-Phase PWM Inverter for High-Performance AC Drives. IEEE Transactions on Industrial Electronics, 45(5), octubre 1998.

Uddin M., Radwan T., Rahman M. Fuzzy-Logic-Controller-Based Cost Effective Four-Switch Three-Phase Inverter-Fed IPM Synchronous Motor Drive System. IEEE Trans. On Industry Applications, 42(1), 2005.

Villada F., Cadavid D. Diagnóstico de fallas en motores de inducción mediante la aplicación de redes neuronales artificiales. Información tecnológica, 18 (2). [en línea]. La Serena 2007 [Fecha de consulta: 10 Agosto 2010]. Disponible en: http://www. scielo.cl/scielo.php?pid=S0718-07642007000200016 \&script=sci_arttext

Von der Beeck M. Development of Logical and Technical Architectures for Automotive Systems. Software and Systems Modeling, 6(2):205-219, 2007. DOI: 10.1007/s102270-006-0022z 


\section{Semblanza de los autores}

Rojas-Molina Adriana. Realizó sus estudios de ingeniería en sistemas computacionales en el Instituto Tecnológico de Querétaro. Ingresó como docente en la Facultad de Informática en el año de 1993. En 2003, finaliza los estudios de maestría en ciencias con línea terminal en instrumentación y control automático en la Facultad de Ingeniería de la Universidad Autónoma de Querétaro. Durante el periodo 2004-2005 fue coordinadora de posgrado en la Facultad de Informática. En el año 2006 ingresó al doctorado en ingeniería de la Universidad Autónoma de Querétaro. Actualmente se encuentra finalizando su tesis doctoral sobre calidad en desarrollo de prototipos electrónicos producidos por centros de investigación y pequeñas empresas.

Herrera-Ruiz Gilberto. Realizó los estudios de ingeniería en sistemas electrónicos en el Instituto Tecnológico de Estudios Superiores de Monterrey, donde finalizó la maestría en ingeniería eléctrica en 1989. En 1992, finalizó los estudios doctorales en la Academia de Ciencias de Hungría y obtuvo una beca por parte de la Agencia de Ciencia y Tecnología de Japón (STA) para realizar una estancia posdoctoral en el Instituto de Ingeniería Mecánica en Tsukuba, Japón, donde laboró bajo la supervisión del doctor Kazuo Mori. En el año 1998, ingresó a la Universidad Autónoma de Querétaro como profesor-investigador y en el año 2004 recibió el Premio ADIAT a la innovación tecnológica. Actualmente se desempeña como director de la Facultad de Ingeniería, de la UAQ.

Castañeda-Miranda Rodrigo. Es doctor en ingeniería por la Universidad Autónoma de Querétaro, se desempeñó como docente en la División de Estudios de Posgrado de la UAQ en los programas de maestría y doctorado de la Facultad de Ingeniería. Ha trabajado en varios proyectos de investigación como director y colaborador, varios de ellos para CONACYT y ALO-USAID. Obtuvo el Premio Nacional a la Innovación Tecnológica 2004 y Premio Internacional INTEL 2005. Actualmente se desempeña como rector de la Universidad Politécnica del Sur de Zacatecas.

Terrazas-Lara Antonio. Realizó los estudios de ingeniería en electrónica en el Instituto Tecnológico de Chihuahua. Laboró en API Análisis Predictivo Industrial S.A. de C.V. donde desarrolló un Software de análisis de vibraciones durante el periodo 2008-2010. Actualmente labora en la empresa Averna Guadalajara como desarrollador de software, ha realizado proyectos empleando FPGA en tiempo real bajo plataforma de desarrollo de National Instruments.

Flores-Chaparro Javier Hissarlik. Estudió ingenieria en electronica con la especialidad de instrumentacion y control en el Instituto Tecnologico de Chihuahua. Trabajó como ingeniero de campo en la automatizacion de sistemas de riego y sistemas de seguridad en la industria privada en 2009. Actualmente se desempeña como ingeniero de aplicaciones en el área de aseguranza de calidad en la compañía Leviton de Mexico Planta Camargo, desde 2010 a la fecha.

Barón-Rodríguez Benjamín. Estudió ingeniero en electrónica, área terminal en instrumentación y control en la Facultad de Ingeniería, Universidad Autónoma de Baja California. Trabajó como auxiliar técnico en el proyecto Parque Eólico La Rumorosa I, en la Comisión Estatal de Energía de Baja California durante 2008 y 2009. Actualmente se desempeña como ingeniero de Software para la compañía Bollseye Inc. de 2010 a la fecha.

Chaparro-Sánchez Ricardo. Realizó sus estudios en ingeniería electrónica en el Instituto Tecnológico de Querétaro. Ingresó como docente a la Facultad de Ingeniería en el año 1996. En el año 2002, terminó los estudios de maestría en ciencias con línea terminal en instrumentación y control automático en la Facultad de Ingeniería de la Universidad Autónoma de Querétaro. En el año 2005, ingresó al doctorado en ciencia y tecnología, del PICYT. Actualmente se encuentra finalizando su tesis doctoral en propuesta de una nariz electrónica con control difuso para la detección de aromas. 